\title{
ARTICLE OPEN \\ Rictor/mTORC2 involves mitochondrial function in ES cells derived cardiomyocytes via mitochondrial Connexin 43
}

\author{
Jia-dan Wang ${ }^{1,2}$, Ying Shao ${ }^{1}$, Dan Liu', Nuo-ya Liu' and Dan-yan Zhu ${ }^{1}$
}

\begin{abstract}
Rictor is a key component of the mammalian target of rapamycin complex 2 (mTORC2) and is required for Akt phosphorylation (Ser473). Our previous study shows that knockdown of Rictor prevents cardiomyocyte differentiation from mouse embryonic stem (ES) cells and induces abnormal electrophysiology of ES cell-derived cardiomyocytes (ESC-CMs). Besides, knockdown of Rictor causes down-expression of connexin 43 (Cx43), the predominant gap junction protein, that is located in both the sarcolemma and mitochondria in cardiomyocytes. Mitochondrial $\mathrm{Cx} 43(\mathrm{mtCx} 43)$ plays a crucial role in mitochondrial function. In this study, we used the model of cardiomyocyte differentiation from mouse ES cells to elucidate the mechanisms for the mitochondrial damage in ESCCMs after knockdown of Rictor. We showed swollen and ruptured mitochondria were observed after knockdown of Rictor under transmission electron microscope. ATP production and mitochondrial transmembrane potential were significantly decreased in Rictor-knockdown cells. Furthermore, knockdown of Rictor inhibited the activities of mitochondrial respiratory chain complex. The above-mentioned changes were linked to inhibiting the translocation of Cx43 into mitochondria by knockdown of Rictor. We revealed that knockdown of Rictor inactivated the mTOR/Akt signalling pathway and subsequently decreased HDAC6 expression, resulted in Hsp90 hyper-acetylation caused by HDAC6 inhibition, thus, inhibited the formation of Hsp90-Cx43-TOM20 complex. In conclusion, the mitochondrial Cx43 participates in shRNA-Rictor-induced mitochondrial function damage in the ESC-CMs.
\end{abstract}

Keywords: Rictor/mTORC2; embryonic stem cell; mitochondria; Connexin43; cardiomyocyte differentiation

Acta Pharmacologica Sinica (2021) 42:1790-1797; https://doi.org/10.1038/s41401-020-00591-3

\section{INTRODUCTION}

The heart is the first functional organ formed during embryonic development [1]. And a high mitochondrial density is crucial to meet the energy demands during embryo cardiac development. The mammalian target of rapamycin complex 2 (mTORC2) pathway was shown to participate in protecting the heart from ischaemic injury, cell proliferation and differentiation $[2,3]$. Furthermore, mTORC2 regulates mitochondrial reactive oxygen species (ROS) and mitochondrial respiration [4]. Rictor is a core component of mTORC2 [5]. Embryos that lacked Rictor exhibited growth arrest and died at E11.5 [6]. In addition, Rictor deletion in embryonic heart tissue was revealed to contribute to heart defects [7].

Mouse embryonic stem (ES) cells differentiated into cardiomyocytes in vitro serve as a suitable model to find new targets for regulating cardiomyocyte formation and to study embryonic cardiac development [8]. Recent studies have demonstrated that mitochondria affect cardiomyocyte differentiation via altering states of mitochondrial permeability transition pore opening [1, 9], mitochondrial fusion [10], mitochondrial DNA transcription factors [11], and mitochondrial reactive ROS [12]. Our previous study showed that knockdown of Rictor inhibited mouse ES cell differentiation into cardiomyocytes. The differentiated cardiomyocytes exhibited irregular myofilaments and disordered electrophysiological activity [13]. In addition, we found that the structure of the mitochondria-endoplasmic reticulum membrane was damaged after knockdown of Rictor, which inhibited the release of calcium from the endoplasmic reticulum to mitochondria [14]. Thus, we speculated that Rictor affects the cardiomyocyte differentiation of ES cells by regulating mitochondrial function.

Connexin $43(\mathrm{C} \times 43)$, the predominant gap junction protein, is mainly localized in the sarcolemma but is also found in the mitochondria and binds heat shock protein 90 (Hsp90) and Tom20 [15-17]. Mitochondrial Cx43 ( $m t C x 43$ ) forms a hemichannel in the mitochondrial inner membrane, regulating mitochondrial potassium uptake [18], ROS generation [19], and energy metabolism [20]. Mitochondrial Cx43 hemichannels were shown to contribute to mitochondrial calcium homoeostasis and cell injury/death in the heart [21]. Studies have shown that $\mathrm{mtCx} 43$ specifically affects the activity of respiratory chain enzyme complex I [22]. Our previous study found that knockdown of Rictor led to decreased expression of $\mathrm{Cx} 43$ in gap junctions in ES cell-derived cardiomyocytes (ESC-CMs) [13]. We speculated that $\mathrm{mtC} \times 43$ was involved in regulating mitochondrial function by Rictor, further influencing energy metabolism, leading to repression of the cardiomyocyte differentiation of ES cells.

The activity of Akt can be regulated by Rictor through Akt phosphorylation (Ser473) [23]. It has been reported that HDAC6 is regulated by the PTEN/Akt/mTOR pathway [24]. Furthermore, the acetylation of Hsp90, mediated by HDAC6, has been shown to be

${ }^{1}$ Institute of Pharmacology and Toxicology, Zhejiang University, Hangzhou 310058, China and ${ }^{2}$ Xiaoshan Traditional Chinese Medical Hospital, Hangzhou 311201, China Correspondence: Dan-yan Zhu (zdyzxb@zju.edu.cn)

These authors contributed equally: Jia-dan Wang, Ying Shao

Received: 21 November 2019 Accepted: 27 September 2020

Published online: 5 February 2021 
involved in the mitochondrial transport of proteins [25]. In addition, Cx43 transport to the mitochondrial inner membrane was found to be dependent on the Hsp90-TOM20 system. However, whether knockdown of Rictor contributed to mtCx43 expression by downregulating HDAC6 and the mechanism of Rictor-regulated mitochondrial function in ESC-CMs remained unclear.

In this study, a model of cardiomyocyte differentiation in mouse ES cells was employed to investigate the mechanism of mitochondrial damage in ESC-CMs after the knockdown of Rictor.

\section{MATERIALS AND METHODS}

Cell culture and cardiomyocyte differentiation

D3 mouse ES cells (American Type Culture Collection, VA, USA) were maintained as previously described $[26,27]$. Briefly, mouse ES cells ( 900) were cultured in a 30- $\mu \mathrm{L}$ hanging droplet for $3 \mathrm{~d}$ in differentiation medium containing DMEM (Gibco, Life Technologies, WA, USA) with $20 \%$ FBS (Gibco), $0.1 \mathrm{mmol} \cdot \mathrm{L}^{-1} \beta$-mercaptoethanol (Sigma-Aldrich, MO, USA), and 1\% NEAAs (Gbico) to form embryoid bodies (EBs). On d 3, the EBs were transferred to Petri dishes and floated for an additional $2 \mathrm{~d}$. Then, on $\mathrm{d} 5$, the EBs were individually plated onto cell culture plates for an additional $3 \mathrm{~d}$.

Infection with short hairpin RNA (shRNA) targeting Rictor and overexpressing $\mathrm{mtC} \times 43$

Lentiviruses containing Rictor shRNA and control shRNA and lentiviruses contain $m t C x 43$ were used to infect mouse ES cells. Lentiviruses contain Rictor or control shRNA was used in the group of shRNA-Con and shRNA-Rictor respectively. In the group of shRNA-Rictor $+m t C x 43$, lentiviruses contain $m t C x 43$ and lentiviruses contain Rictor shRNA were used together in order to knockdown of Rictor and over express mtCx43. shRNA-Rictor and shRNA-Con were ordered from Genepharma Company (Shanghai, China). Mouse $m t C x 43$ labeled with RFP was ordered from Hanheng Biotechnology Company (Shanghai, China).

shRNA-Rictor: 5'-GCCAGTAAGATGGGAATCATT-3',

shRNA-Con: 5'-TTCTCCGAACGTGTCACGTTC-3',

and sequence target mitochondria: 5'-ATGTCCGTCCTGACGCCG CTGCTGCTGCGGGGCTTGACAGGCTCGGCCCGGCGGCTCCCAGTGCC GCGCGCCAAGATCCATTCGTT-3'.

Briefly, $1 \times 10^{5}$ mouse ES cells per well were seeded into 12-well plates. The cells were infected with an aliquot of lentivirus to achieve a multiplicity of infection of 50 PFU/cell $4 \mathrm{~h}$ later. After infection for $24 \mathrm{~h}$, cells were harvested for EB formation.

\section{Isolation of ESC-CMs}

On $d 5+3$, the beating areas of the EBs were dissociated by gentle pipetting with a glass pipette with an internal diameter of 200-300 $\mu \mathrm{m}$. Then, the cell clusters were digested into individual cells with Accutase enzyme solution (Life Technologies) for $30 \mathrm{~min}$ at $37^{\circ} \mathrm{C}$. The isolated cells were collected for further analyses [28].

\section{Western blot analysis}

Cell lysates were prepared in Western blot lysis buffer (Beyotime, Shanghai China). Mitochondrial/cytoplasmic proteins were obtained from samples with a mitochondrial protein extraction kit (Beyotime). Samples were coimmunoprecipitated using Protein $A+G$ agarose beads (Beyotime) according to the manufacturer's instructions. Western blotting was performed as previously reported $[26,27]$. Antibodies against thefollowing proteins were used: GAPDH, a-actinin (Sigma Aldrich), Akt1/2/3 (Ser 473), Akt1/2/ 3, Rictor, VDAC1 (Santa Cruz, TX, USA), cytochrome c, Oct4, mTOR, p-mTOR (Ser2481), HDAC6, TOM20 (Cell Signalling Technology, MA, USA), c-TNT, Connexin 43, Rictor, Hsp90, SIN1, G protein beta subunit like, and acetyl-lysine (Abcam, MA, USA). The membranes were incubated with horseradish peroxidase (HRP)-conjugated antibodies (Lianke, Hangzhou, China). All data analyses were carried out by using ImageJ software.

Flow cytometry analysis

EBs obtained on d $5+3$ of differentiation were harvested and digested into single cells with Accutase enzyme solution (Life Technologies). Cells were fixed with $4 \%$ paraformaldehyde for $1 \mathrm{~h}$ and then blocked with $5 \%$ bovine serum albumin (BSA, Sigma Aldrich) for another $1 \mathrm{~h}$ at room temperature. After that, the cells were incubated with monoclonal anti-a-actinin antibody (Sigma Aldrich, 1:400) or monoclonal anti-c-TNT antibody (Abcam, 1:400) overnight. After being washed with PBS three times, the cells were incubated with DyLight 488-conjugated anti-mouse IgG (1:400) at $4{ }^{\circ} \mathrm{C}$ for $1 \mathrm{~h}$. Then, a total of $1 \times 10^{4}$ cells were suspended in $0.5 \mathrm{~mL}$ of $1 \%$ BSA and analyzed by FACScan flow cytometry (Becton Dickinson, NJ, USA) $[26,29]$.

Measurement of mitochondrial membrane potential $\left(\Delta \Psi_{\mathrm{m}}\right)$ The $\Delta \Psi_{\mathrm{m}}$ of ESC-CMs was measured as previously described [26] following the manufacturer's instructions. ESC-CMs on $\mathrm{d} 5+3$ were plated on 48 -well plates at a density of $3 \times 10^{4}$ cells per well. After $48 \mathrm{~h}$, the cells were incubated with $2 \mu \mathrm{g} \cdot \mathrm{mL}^{-1} \mathrm{JC}-1$ dye (Beyotime) at $37^{\circ} \mathrm{C}$ in the dark for $30 \mathrm{~min}$. Then, the cells were washed with washing buffer, and images were obtained with a Leica DMI3000B microscope (Leica Microsystems, Wetzlar, Germany).

Cellular ATP assay

ESC-CMs on d $5+3$ were assessed with an ATP bioluminescence assay kit (S0026, Beyotime) following the manufacturer's instructions. All the data were standardized to the control group.

Immunofluorescence analysis

Immunofluorescence analysis of ESC-CMs on d $5+3$ was performed as previously described [26, 27]. Cells were incubated with MitoTracker or ER-Tracker (Life Technologies) for 30 min in the dark and then fixed with $4 \%$ paraformaldehyde for $30 \mathrm{~min}$. The cells were then blocked with $5 \%$ BSA for $1 \mathrm{~h}$ at room temperature. After that, the cells were incubated with antibody against Cx43 (Abcam, 1:400) or Rictor (Abcam, $1: 400$ ) at $4{ }^{\circ} \mathrm{C}$ overnight. Next, the cells were washed with PBS three times and then incubated with Alexa Fluor 488-conjugated anti-rabbit IgG (Lianke, 1:400) or DyLight 650conjugated anti-rabbit IgG (Invitrogen, CA, USA, 1:400) antibody at $4{ }^{\circ} \mathrm{C}$ for $2 \mathrm{~h}$. The cells were observed under an Olympus FV3000 confocal microscope (Olympus, Hertfordshire, UK).

\section{Transmission electron microscopy}

ESC-CMs on d $5+3$ were fixed with $2.5 \%$ glutaraldehyde at $4{ }^{\circ} \mathrm{C}$ for $2 \mathrm{~h}$ and $1 \%$ osmium tetroxide for $1.5 \mathrm{~h}$. Then, the cells were dehydrated with gradient ethanol (30\%, 50\%, 70\%, 80\%, $90 \%$ and $100 \%)$ and pure acetone. The cells were embedded in pure embedding agent and sectioned at a thickness of $70-90 \mathrm{~nm}$. Then, the samples were dyed with lead citrate and uranyl acetate solutions and observed under a transmission electron microscope [26].

Respiratory chain enzyme assays

The activities of individual enzymes in complexes I, III, IV and V were measured following the manufacturer's instructions. Complex I activity was measured by monitoring NADH oxidation at $340 \mathrm{~nm}$. Complex III and IV activities were measured by monitoring the reduction andoxidation, respectively, of cytochrome $c$ at $550 \mathrm{~nm}$. Citrate synthase was assayed by monitoring 5,5'-dithiobis (2-nitrobenzoid) reduction at $412 \mathrm{~nm}$.

\section{Statistics analysis}

Data are expressed as the mean values \pm standard deviations. At least 3 independent experiments were performed as replicates. 
Statistical analyses were performed by $t$ test when two groups were compared. A value of $P<0.05$ indicated a significant difference.

\section{RESULTS}

Knockdown of Rictor impaired mitochondrial structure and function in ESC-CMs

The level of Rictor during the cardiomyocyte differentiation of mouse ES cells was upregulated, as shown by examination by Western blot analysis (Fig. 1a). After the transfection of shRNARictor lentivirus, the cell viability and pluripotency of the ESCs were not different from those of the shRNA-control group (Fig. 1b, c). Knockdown of Rictor inhibited cardiomyocyte differentiation. After the knockdown of Rictor, the proportion of cells positive for a-actinin (a cardiomyocyte biomarker) on d $5+3$ was significantly decreased to $11.8 \% \pm 1.0 \%$ compared to that in the control group $(19.9 \% \pm 1.2 \%)$, as shown by flow cytometry analysis (Fig. 1d). The Western blot results also showed that the expression level of a-actinin was markedly reduced in Rictorknockdown cells (Fig. 1e). In addition, the presence of Troponin T in cardiomyocytes (CTNT) typically indicates a relatively late phase of differentiation. We detected the expression of cTNT by Western blot analysis and flow cytometry. The results showed no significant difference in cTNT levels between the shRNA-Con and shRNA-Rictor groups on days $5+3$, while on day $5+5$, the expression of cTNT was decreased in the shRNA-Rictor group compared to the shRNA-Con group (Fig. 1f, g). These results demonstrated that knockdown of Rictor prevented cardiomyocyte differentiation from mouse ES cells. Cardiomyocytes with a purity of $83.4 \% \pm 3.7 \%$ (Supplementary Fig. 1) were used to explore mitochondrial function in ESC-CMs. Transmission electron microscopy showed that the mitochondria in shRNA-Rictortreated ESC-CMs appeared swollen and contained more vacuoles (Fig. $1 \mathrm{~h}$ ). We further examined the $\Delta \Psi_{\mathrm{m}}$ by $\mathrm{JC}-1$ staining and found that ESC-CMs in the Rictor-knockdown group exhibited a lower ratio of red/green fluorescence intensity than those in the control group (Fig. 1i). Flow cytometry analysis also showed that the ratio of green $(\mathrm{FL} 1-\mathrm{H}) /$ red $(\mathrm{FL} 2-\mathrm{H})$ fluorescence was significantly increased in Rictor-knockdown cells, indicating a low $\Delta \Psi_{\mathrm{m}}$ (Fig. 1j). The intracellular ATP level on $d 5+3$ was significantly decreased in shRNA-Rictor cells compared to the control group (Fig. 1k). To determine whether shRNA-Rictor transfection triggered apoptosis, the release of cytochrome $c$ from the mitochondria was measured by Western blot analysis. The mitochondrial and cytoplasmic cytochrome $c$ contents were not significantly different between the two groups (Fig. 1l).

$\mathrm{mtC} 43$ is involved in regulating mitochondrial function in ESCCMs, as shown by shRNA-Rictor transfection

The results of Western blot assays showed that total Cx43 and $\mathrm{mtC} 43$ were significantly reduced in shRNA-Rictor cells (Fig. 2a). The ratio of Cx43 (green) and MitoTracker (red) (Fig. 2b) colocalization was significantly downregulated by shRNA-Rictor transfection compared with that in the control group. An increased cardiomyocyte differentiation efficiency was found in cells transfected with both shRNA-Rictor and $m t C x 43$. The proportion of a-actinin-positive cells on d $5+3$ was increased to $18.3 \% \pm 1.1 \%$ compared with $13.6 \% \pm 1.0 \%$ in the shRNA-Rictor group on $\mathrm{d} 5+3$, as shown by flow cytometry analysis (Fig. 2c). In addition, flow cytometry analysis showed that the proportion of cTNT-positive cells on d $5+5$ was increased to $25.6 \% \pm 0.8 \%$ compared with $18.3 \% \pm 1.1 \%$ in the shRNA-Rictor group (Fig. $2 \mathrm{~d}$ ). To investigate whether Rictor regulates the function of mitochondria via $\mathrm{mtCx} 43$, we overexpressed $\mathrm{mtC} \times 43$ in ES cells. Overexpression of $\mathrm{mtC} \times 43$ mitigated the shRNA-Rictor-induced decrease in intracellular ATP production and $\Delta \Psi_{\mathrm{m}}$ (Fig. 2e, f).
Rictor-mediated regulation of the mitochondrial respiratory chain via $\mathrm{mtCx} 43$

Assays to detect the activities of respiratory chain enzymes showed that the activities of complex I, IV, and V were markedly reduced in shRNA-Rictor cells (Fig. 3a, c, d), while complex III activity was not significantly changed (Fig. 3b). Decreases in the activities of complexes I and IV were significantly reversed in ESCCMs transfected with shRNA-Rictor and $m t C x 43$ compared with the shRNA-Rictor group (Fig. 3a, c). Overexpression of $\mathrm{mtCx} 43$ slightly attenuated the decrease in complex $\mathrm{V}$ activity caused by the knockdown of shRNA-Rictor, but this effect did not reach statistical significance (Fig. 3d).

The mechanism by which Rictor regulates the translocation of Cx43 to mitochondria

Cx43 is translocated to the inner mitochondrial membrane through the Hsp90-dependent TOM20 pathway [30]. Knockdown of Rictor led to a significant decrease in the interactions of Hsp90 and TOM20 with Cx43 in ESC-CMs (Fig. 4a). Decreased levels of pHDAC6 and increased Hsp90 acetylation were observed in Rictorknockdown cells compared to the control group (Fig. 4b, c). Furthermore, knockdown of Rictor destroyed the integrity and activity of mTORC2, as evidenced by diminished levels of $p$ mTOR $^{\text {Ser2481, SIN1 and p-Akt }}{ }^{\text {Ser473 }}$ (Fig. 4d).

\section{DISCUSSION}

mTORC2 was reported to play important roles in the development of embryonic and extraembryonic tissues. The formation of EBs from human amniotic fluid stem cells was found to depend on the activities of mTORC2 [31]. The mTORC2 consists of Rictor, mTOR, $\mathrm{G} \beta \mathrm{L}$ and SIN1. Among these, Rictor is the core of the mTORC2 complex [32]. Embryos with Rictor knockdown showed growth arrest at E9.5, death at E13.5 and defective placental development [6]. Moreover, Rictor was confirmed to be crucial to embryonic heart development [7]. Similarly, in our study, we found that the protein expression of Rictor increased during the process of cardiomyocyte differentiation of mouse ES cells. Knockdown of Rictor significantly reduced cardiomyocyte differentiation efficiency, suggesting that Rictor/mTORC2 had a positive effect on cardiomyocyte differentiation.

In cardiomyocytes, the phosphorylation of Akt at Ser473 is modulated by the upstream regulator $\mathrm{MTORC2}$ [7]. In addition, the PI3K-Akt-mTOR pathway regulates the metabolism and proliferation of cardiomyocytes. In our study, we found that knockdown of Rictor destroyed the integrity of mTORC2 and then decreased the phosphorylation of Akt at Ser473. mTORC2 modulates mitochondrial function via Akt, including its increase of ATP production and mitochondrial membrane potential [33]. Cardiac mitochondria play a crucial role in the maintenance of cellular bioenergetics and energy metabolism, as they provide ATP to meet the energy demand in cardiomyocyte differentiation and excitationcontraction coupling [34]. Further evidence has revealed that $\mathrm{mtC} \times 43$ modulates mitochondrial function in different ways, such as its involvement in the homoeostasis of iron, calcium and potassium and reactive oxygen species generation [21]. In our study, we found that upon the knockdown of Rictor in ESC-CMs, the translocation of $\mathrm{Cx} 43$ into mitochondria was decreased, and the mitochondrial structure and function were damaged. However, when we overexpressed mtCx43 in Rictor-knockdown cells, mitochondrial dysfunction and the changes in the cardiac differentiation rate were ameliorated, which indicated that Rictor modulates mitochondrial function via $\mathrm{mtC} \times 43$. Furthermore, it was reported that the expression of $\mathrm{mtC} \times 43$ is modulated by Akt activation. The expression of $\mathrm{mtCx} 43$ in the rat cortex was reduced by treatment with the PI3K/Akt pathway inhibitor LY 294002 (LY) [35]. 
a

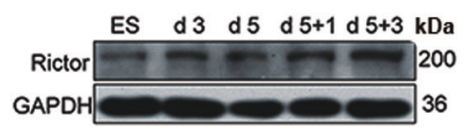

d

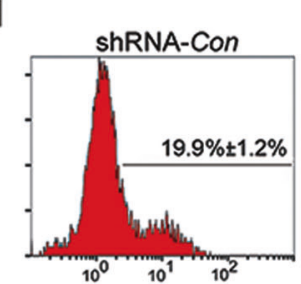

f

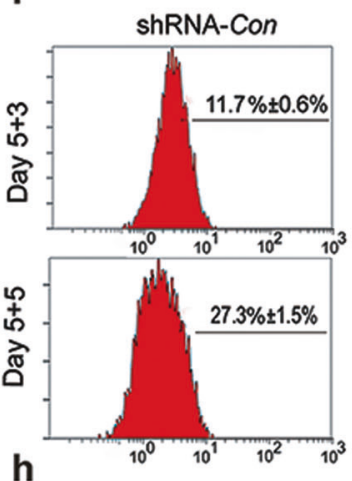

h
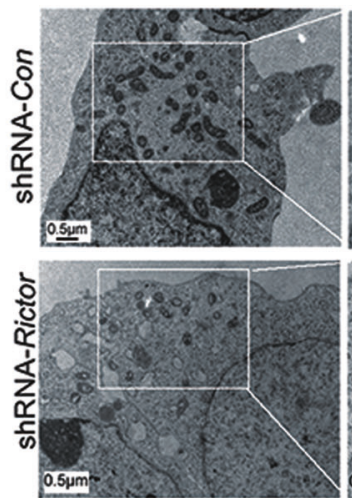

I
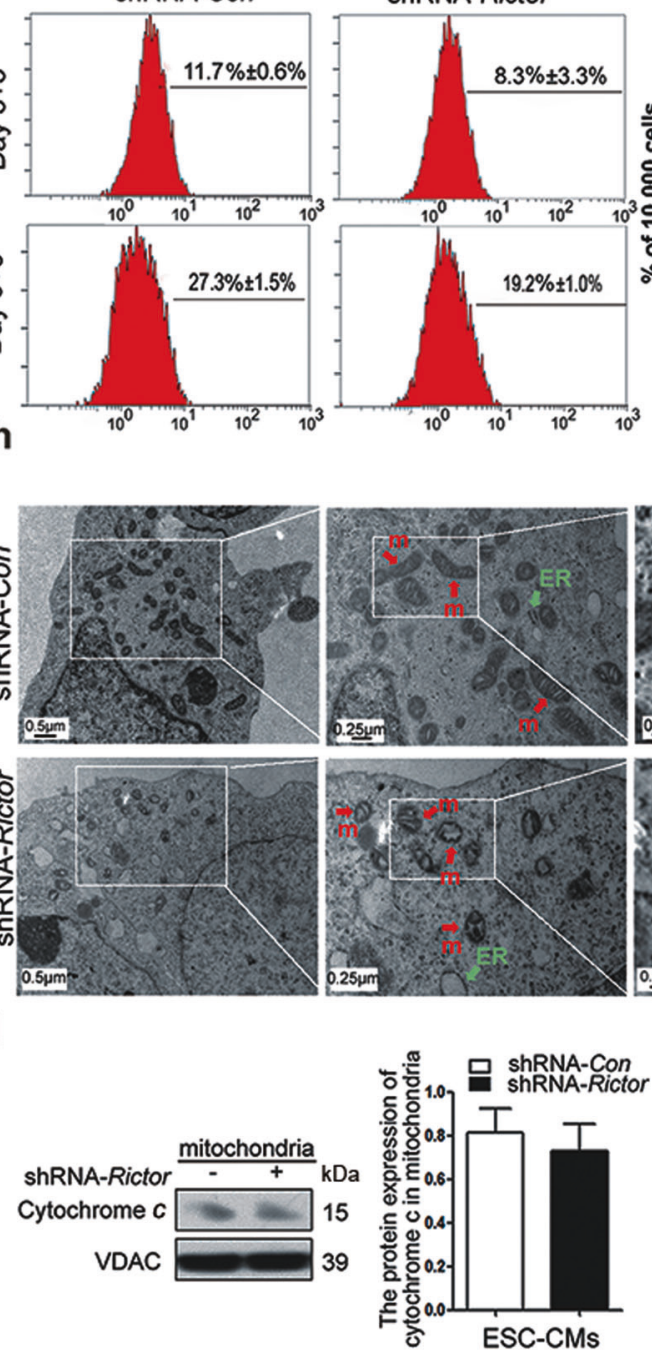

b

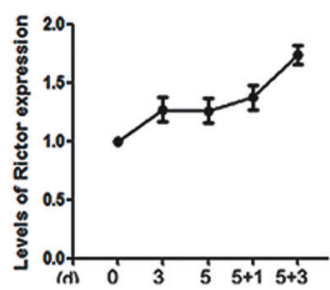

C

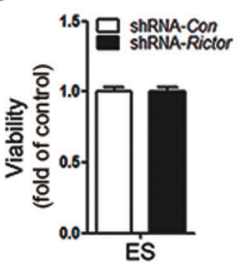

ShRNA-Rictor $\frac{\mathrm{ES}}{-} \mathrm{kDa}$ Rictor 200

OCT4 GAPDH 36
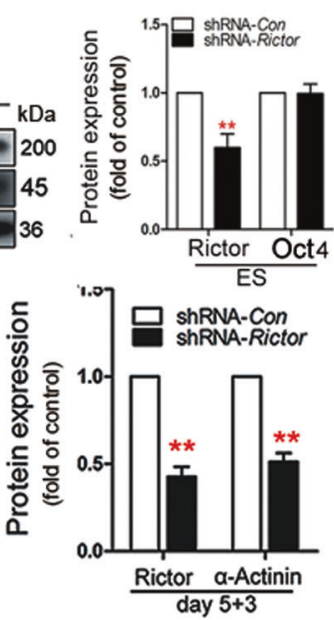

g

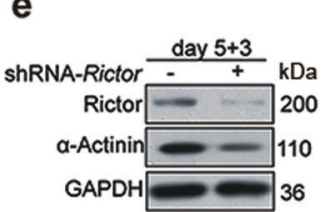

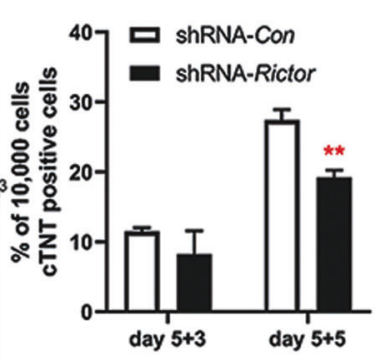

day $5+3$ day $5+5$
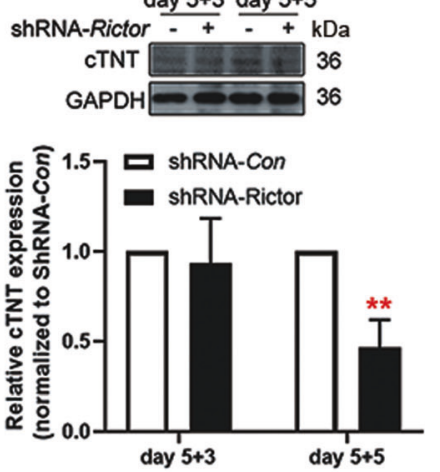

i
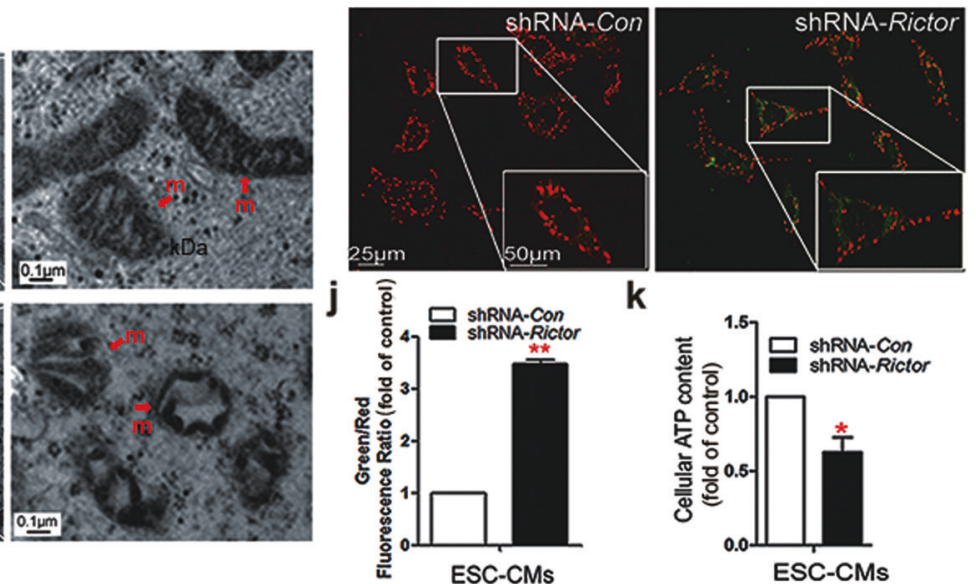

k
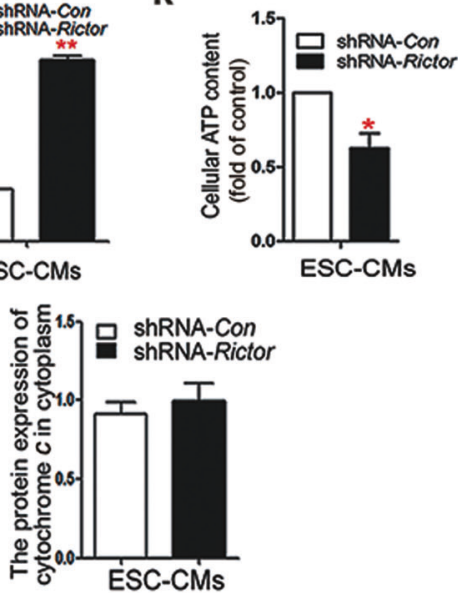

Fig. 1 Knockdown of Rictor inhibited cardiomyocyte differentiation and impaired the mitochondrial structure and function in ESC-CMs. $\mathbf{a}$ The expression of Rictor/mTORC2 during cardiomyocyte differentiation. $\mathbf{b}$ The cell viability of ES cells after transfection with shRNA lentivirus. c The expression of Rictor and Oct4 after transfection with shRNA lentivirus. d Proportions of $\alpha$-actinin-positive cells in the shRNA-Con and shRNA-Rictor groups on d $5+3$ determined by flow cytometry. e The protein expression of $\alpha$-actinin was evaluated in EBs on d $5+3$. $\mathbf{f}$ The proportions of c-TNT-positive cells in the shRNA-Con and shRNA-Rictor groups on d $5+3$ and $d 5+5$, determined by flow cytometry. $\mathbf{g}$ The protein expression of c-TNT was evaluated in EBs on d $5+3$ and $\mathbf{d} 5+5$. $\mathbf{h}$ The ultrastructure of ESC-CMs after Rictor knockdown. (m: mitochondrion, ER: endoplasmic reticulum). $\mathbf{i}$ The $\Delta \Psi \mathrm{m}$ of ESC-CMs was evaluated with JC- 1 staining. $\mathbf{j}$ The $\Delta \Psi \mathrm{m}$ of ESC-CMs was further assessed by flow cytometry. k Intracellular ATP production by ESC-CMs. I The expression levels of cytochrome $c$ in the mitochondria and cytoplasm were no different between the two groups. $n \geq 3$. ${ }^{*} P<0.05,{ }^{*} P<0.01$ vs shRNA-Con. Bars $=0.1 \mu \mathrm{m}, 0.25 \mu \mathrm{m}, 0.5 \mu \mathrm{m}, 25 \mu \mathrm{m}$, and $50 \mu \mathrm{m}$. 
a

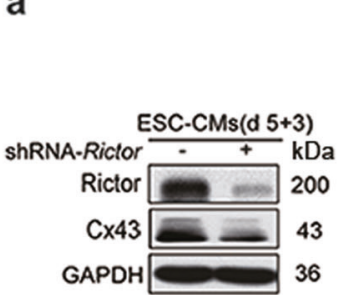

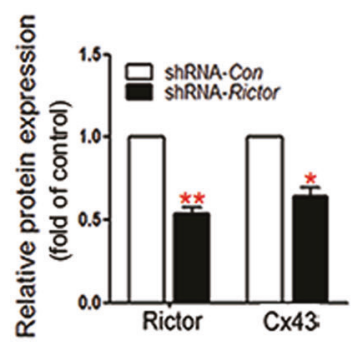
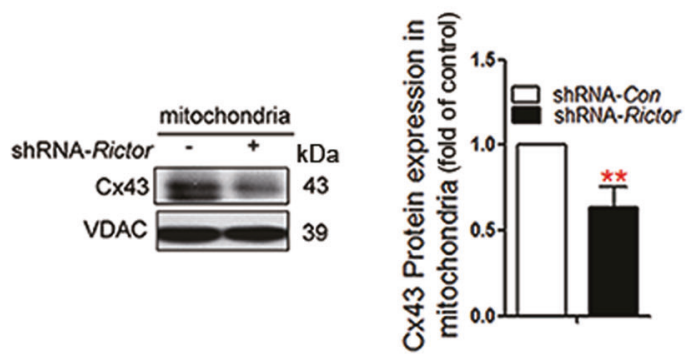

b
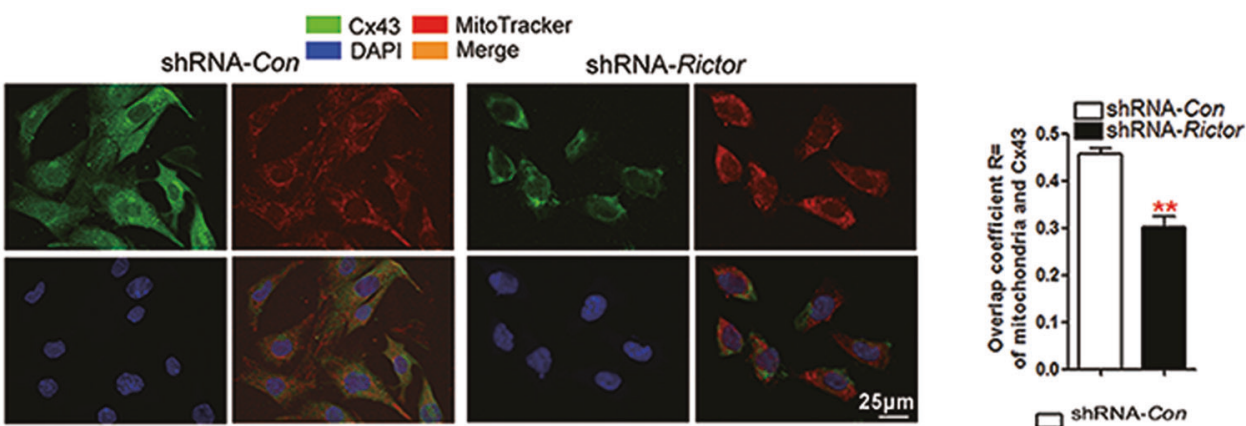

C
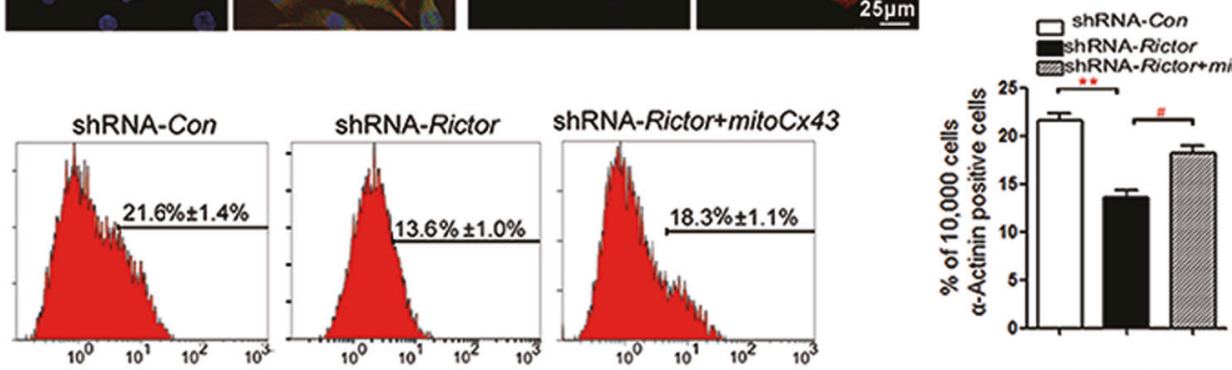

d

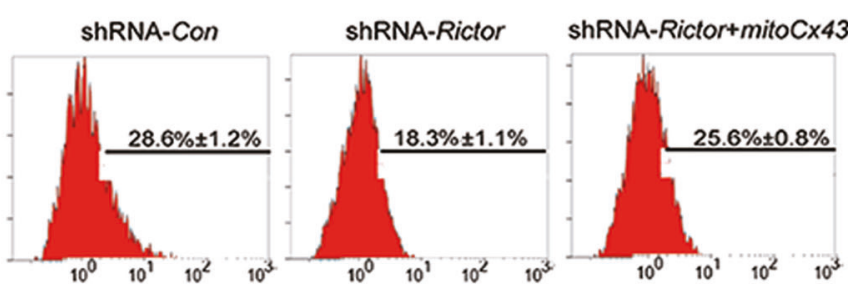

e
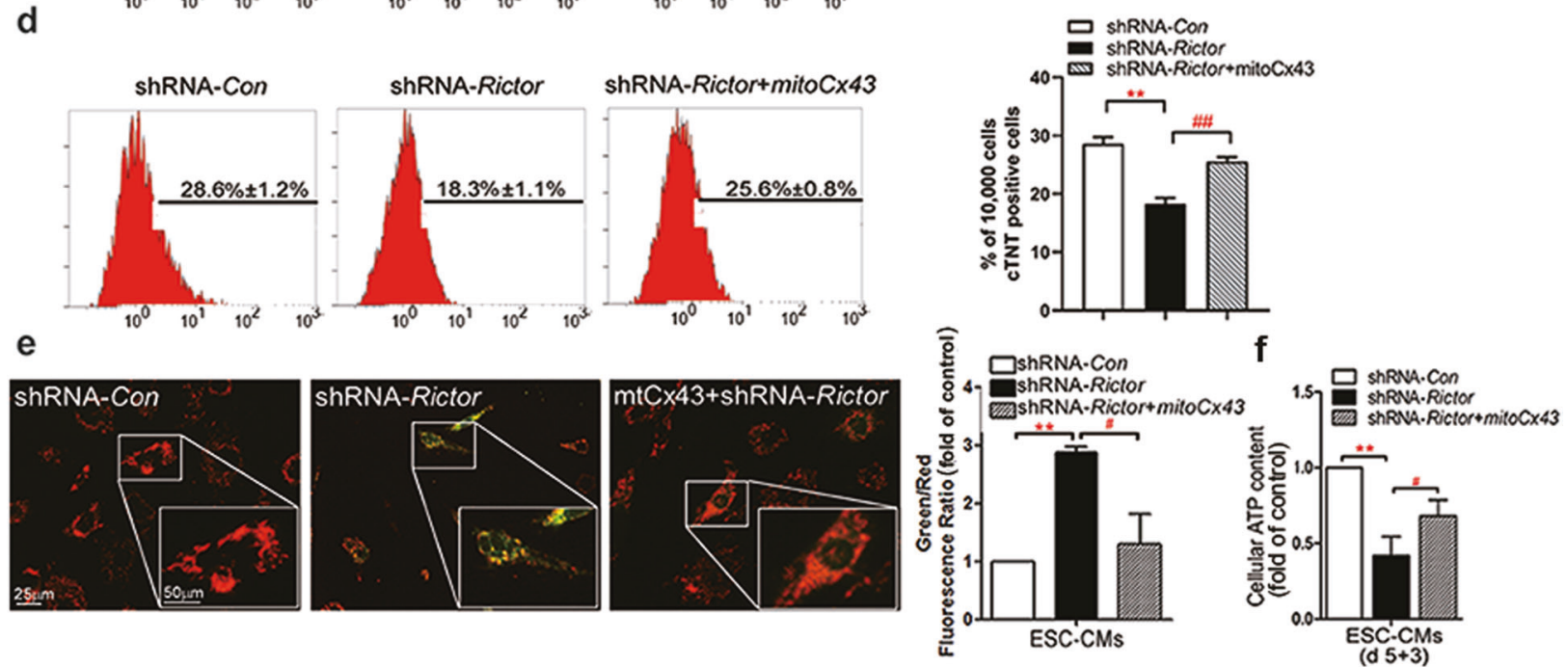

Fig. 2 MtCx43 is involved in the shRNA-Rictor-induced damage to mitochondrial function. a The protein expression levels of $\mathrm{Cx} 43$ in the total cell lysate and mitochondria. b ESC-CMs were double-stained for Cx43 (green) and mitochondria (red). c The proportion of $\alpha$-actinin-positive cells in EBs on d $5+3$ was determined by flow cytometry analysis. $\mathbf{d}$ The proportions of c-TNT-positive cells in EBs on d $5+5$ were determined by flow cytometry analysis. e The $\Delta \Psi \mathrm{m}$ in ESC-CMs were detected by JC- 1 staining and flow cytometry. f Intracellular ATP production was assessed in ESC-CMs at d $5+3 . n \geq 3$. ${ }^{* *} P<0.01$ vs shRNA-Con; ${ }^{\#} P<0.05,{ }^{\# \#} P<0.01$ vs shRNA-Rictor. Bars $=25 \mu \mathrm{m}$ and $50 \mu \mathrm{m}$.

Cx43 transport to the mitochondrial inner membrane is dependent on the Hsp90-TOM20 transport system [30]. In addition, the deacetylation of Hsp90, mediated by HDAC6, has been shown to be involved in the mitochondrial transport of proteins $[36,37]$. In addition, HDAC6 inactivation and knockdown experiments showed that HDAC6 contributes to Hsp90 hyperacetylation and the loss of Hsp90 chaperone activity [38]. Meanwhile, it was revealed that in head and neck squamous cell carcinoma, inhibition of mTOR/Akt pathway signalling inhibits HDAC6 [39, 40]. Consistently, in our study, we found that knockdown of Rictor resulted in inactivation of the mTOR/Akt pathway and subsequently decreased HDAC6 expression in ESCMs. As a result, in ESC-CMs, the increased acetylation of Hsp90 mediated by HDAC6 inhibition disturbed $\mathrm{Cx} 43$ translocation. These results suggested that knockdown of Rictor could interfere with the translocation of $\mathrm{C} \times 43$ from the plasma to mitochondria 

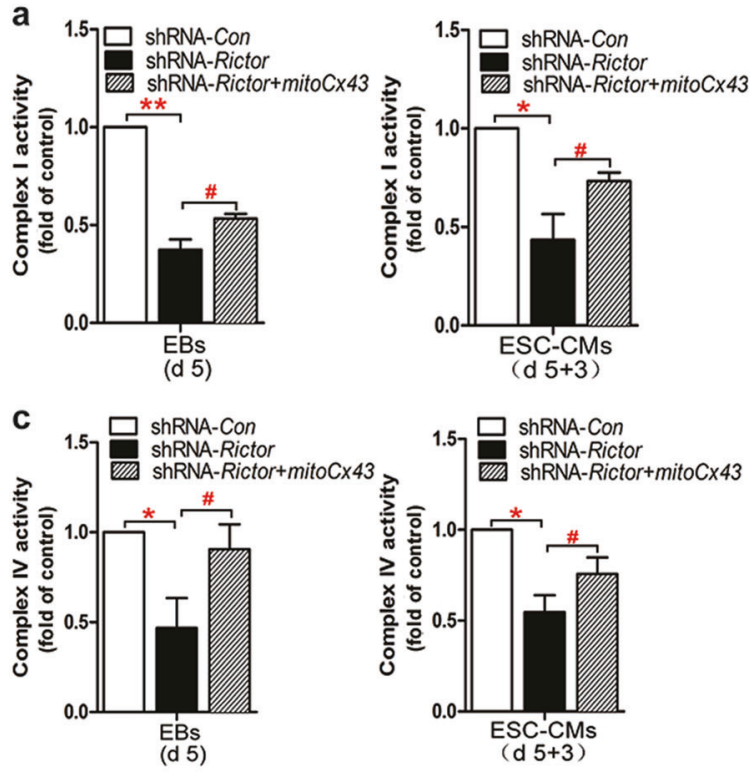

b
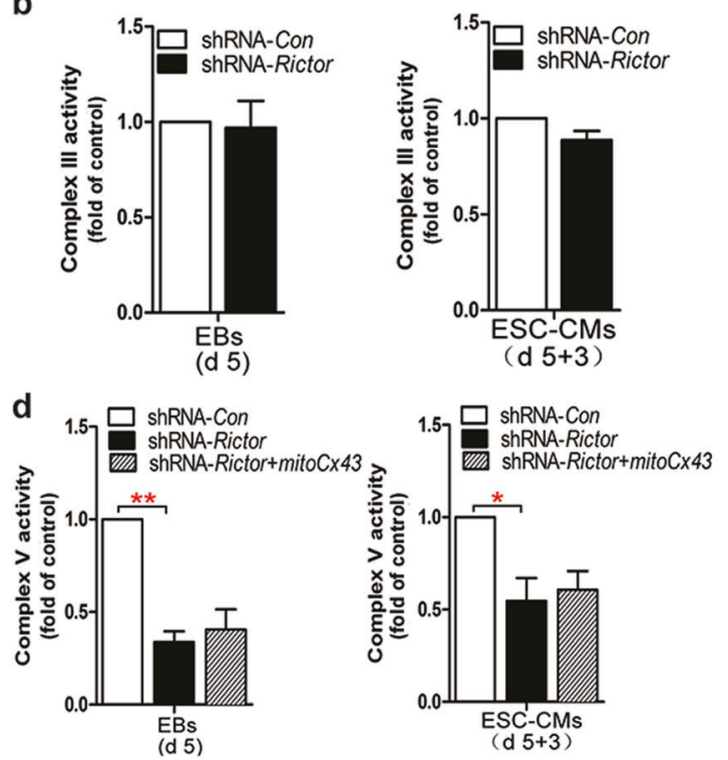

Fig. 3 Rictor-mediated regulation of the mitochondrial respiratory chain via $\mathbf{m t C x} 43$. The activities of enzymes in complexes I (a), III (b), IV (c), and V (d) in Rictor-knockdown ESC-CMs were determined. $n \geq 3$. ${ }^{*} P<0.05,{ }^{* *} P<0.01$ vs shRNA-Con; ${ }^{\#} P<0.05$ vs shRNA-Rictor.
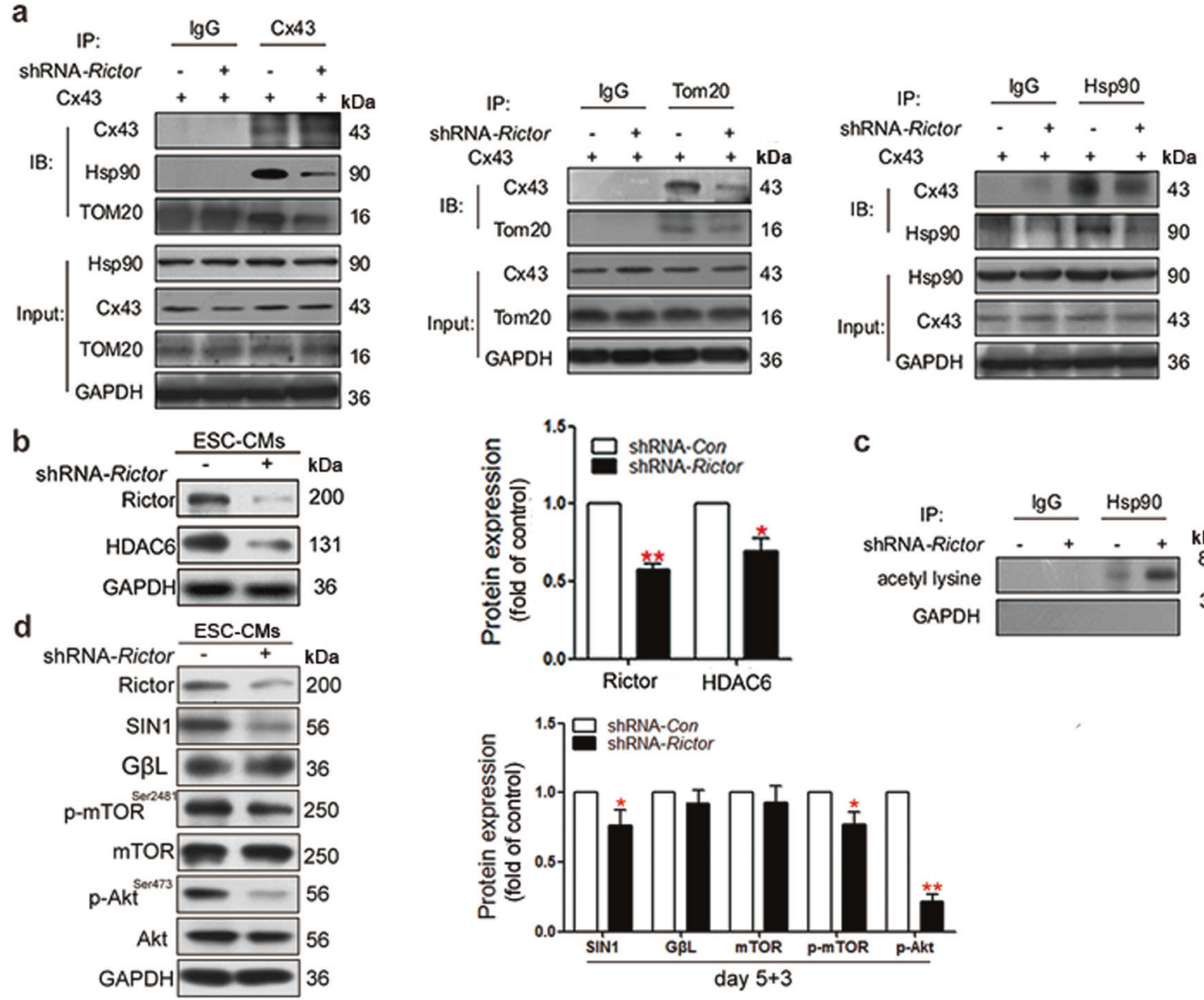

Fig. 4 The mechanism by which Rictor regulates the translocation of Cx43 to mitochondria. a The interaction of Cx43 with the mitochondrial protein import system (Hsp90, TOM20) in ESC-CMs was decreased after knockdown of Rictor. b The protein expression level of HDAC6 in ESC-CMs. c Knockdown of Rictor increased the acetylation of Hsp90 in ESC-CMs. d Knockdown of Rictor decreased the expression of SIN1, p-Akt ${ }^{\text {Ser473, }}$, and p-mTOR ${ }^{\text {Ser2481 }}$ in ESC-CMs. $n \geq 3 .{ }^{*} P<0.05, * * P<0.01$ vs shRNA-Con. 


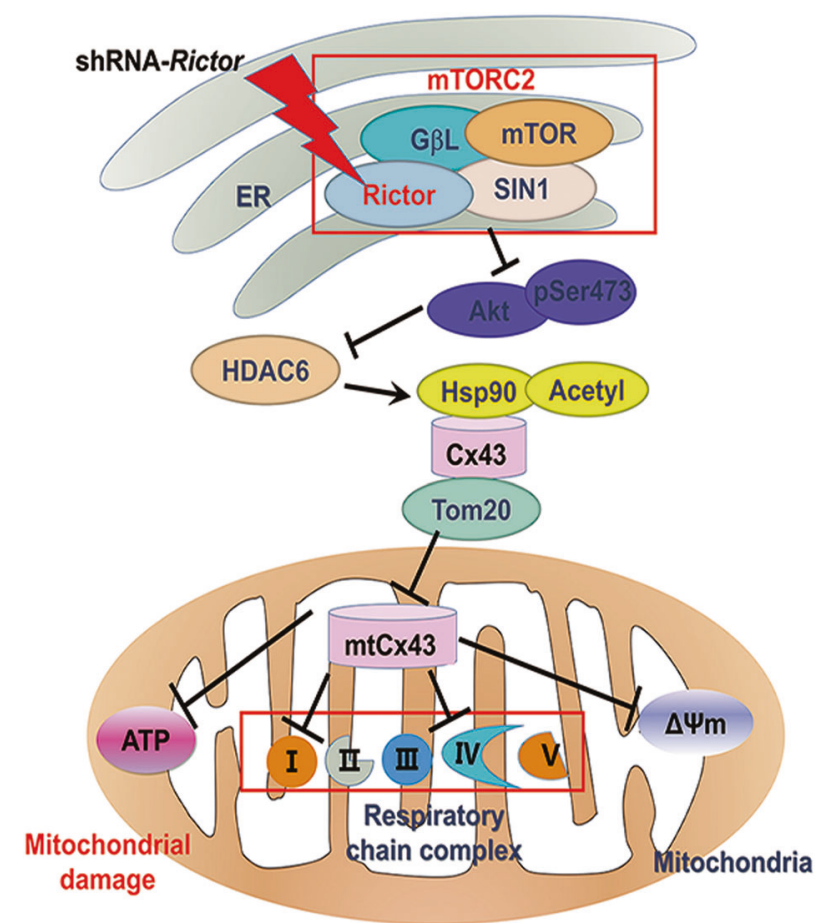

Fig. 5 Schematic representation of the mechanisms by which Rictor regulates mitochondrial function in ESC-CMs.

by decreasing HDAC6 expression. In conclusion, knockdown of Rictor resulted in mitochondrial dysfunction owing to the inhibition of Cx43 translocation to mitochondria. Mechanistically, this effect might have involved inactivation of the mTOR/Akt signalling pathway and a subsequent decrease in Hsp90-Cx43TOM20 formation attributed to Hsp90 hyperacetylation mediated by HDAC6 inhibition due to Rictor knockdown. Therefore, mtCx43 participated in shRNA-Rictor-induced mitochondrial function damage in ESC-CMs, providing an experimental basis for further investigation of the mechanisms of Rictor-regulated cardiomyocyte differentiation (Fig. 5).

\section{ACKNOWLEDGEMENTS}

We thank to the Core Facilities and Center of Electron Microscopy, Zhejiang University School of Medicine for the technical support.

\section{AUTHOR CONTRIBUTIONS}

DYZ: Conception and design, financial support, final approval of manuscript; JDW: Provision of study material, acquisition of data and data analysis, manuscript writing; YS: Performed the part of experiments, manuscript writing; DL and NYL: Partial acquisition of data.

\section{FUNDING}

This work was supported by National Key R\&D Program of China (No. 2017YFE0102200); the National Natural Science Foundation of China (No. 81573426); the State Key Program of National Natural Science Foundation of China (No.81730101), Public Welfare Project of Zhejiang Provincial Science and Technology Department (No. LGF21H310005).

\section{ADDITIONAL INFORMATION}

The online version of this article (https://doi.org/10.1038/s41401-020-00591-3) contains supplementary material, which is available to authorized users.

Competing interests: The authors declare no competing interests.

\section{REFERENCES}

1. Hom JR, Quintanilla RA, Hoffman DL, de Mesy Bentley KL, Molkentin JD, Sheu SS, et al. The permeability transition pore controls cardiac mitochondrial maturation and myocyte differentiation. Dev Cell. 2011;21:469-78.

2. Volkers $M$, Konstandin $M H$, Doroudgar $S$, Toko $H$, Quijada $P$, Din $S$, et al. Mechanistic target of rapamycin complex 2 protects the heart from ischemic damage. Circulation. 2013;128:2132-44.

3. Yano T, Ferlito M, Aponte A, Kuno A, Miura T, Murphy E, et al. Pivotal role of mTORC2 and involvement of ribosomal protein S6 in cardioprotective signaling. Circ Res. 2014;114:1268-80.

4. Vlahakis A, Lopez Muniozguren N, Powers T. Mitochondrial respiration links TOR complex 2 signaling to calcium regulation and autophagy. Autophagy. 2017;13: $1256-7$.

5. Oh WJ, Jacinto E. mTOR complex 2 signaling and functions. Cell Cycle. 2011;10:2305-16.

6. Shiota C, Woo JT, Lindner J, Shelton KD, Magnuson MA. Multiallelic disruption of the rictor gene in mice reveals that mTOR complex 2 is essential for fetal growth and viability. Dev Cell. 2006;11:583-9.

7. Zhao X, Lu S, Nie J, Hu X, Luo W, Wu X, et al. Phosphoinositide-dependent kinase 1 and mTORC2 synergistically maintain postnatal heart growth and heart function in mice. Mol Cell Biol. 2014;34:1966-75.

8. Takahashi T, Lord B, Schulze PC, Fryer RM, Sarang SS, Gullans SR, et al. Ascorbic acid enhances differentiation of embryonic stem cells into cardiac myocytes. Circulation. 2003;107:1912-6.

9. Cho SW, Park JS, Heo HJ, Park SW, Song S, Kim I, et al. Dual modulation of the mitochondrial permeability transition pore and redox signaling synergistically promotes cardiomyocyte differentiation from pluripotent stem cells. J Am Heart Assoc. 2014;3:e000693.

10. Kasahara A, Cipolat S, Chen Y, Dorn GW 2nd, Scorrano L. Mitochondrial fusion directs cardiomyocyte differentiation via calcineurin and Notch signaling. Science. 2013;342:734-7.

11. St John JC, Ramalho-Santos J, Gray HL, Petrosko P, Rawe VY, Navara CS, et al. The expression of mitochondrial DNA transcription factors during early cardiomyocyte in vitro differentiation from human embryonic stem cells. Cloning Stem Cells. 2005;7:141-53.

12. Crespo FL, Sobrado VR, Gomez L, Cervera AM, McCreath KJ. Mitochondrial reactive oxygen species mediate cardiomyocyte formation from embryonic stem cells in high glucose. Stem Cells. 2010;28:1132-42.

13. Zheng B, Wang J, Tang L, Tan C, Zhao Z, Xiao Y, et al. Involvement of Rictor/ mTORC2 in cardiomyocyte differentiation of mouse embryonic stem cells in vitro. Int J Biol Sci. 2017;13:110-21.

14. Shao Y, Wang J, Zhu D. Rictor regulates mitochondrial calcium signaling in mouse embryo stem cell-derived cardiomyocytes. Zhejiang Da Xue Xue Bao Yi Xue Ban Med Sci. 2019;48:65-74.

15. Boengler K. Stimulation of cardiac beta-adrenoceptors targets connexin $43 . \mathrm{Br} J$ Pharmacol. 2009;158:195-7.

16. Boengler K, Schulz R, Heusch G. Connexin 43 signalling and cardioprotection. Heart. 2006;92:1724-7.

17. Duffy HS, Fort AG, Spray DC. Cardiac connexins: genes to nexus. Adv Cardiol. 2006;42:1-17.

18. Miro-Casas E, Ruiz-Meana M, Agullo E, Stahlhofen S, Rodriguez-Sinovas A, Cabestrero A, et al. Connexin43 in cardiomyocyte mitochondria contributes to mitochondrial potassium uptake. Cardiovasc Res. 2009;83:747-56.

19. Heinzel FR, Luo Y, Li X, Boengler K, Buechert A, Garcia-Dorado D, et al. Impairment of diazoxide-induced formation of reactive oxygen species and loss of cardioprotection in connexin 43 deficient mice. Circ Res. 2005;97:583-6.

20. Rodriguez-Sinovas A, Sanchez JA, Gonzalez-Loyola A, Barba I, Morente $M$, Aguilar $\mathrm{R}$, et al. Effects of substitution of $\mathrm{C} \times 43$ by $\mathrm{C} \times 32$ on myocardial energy metabolism, tolerance to ischaemia and preconditioning protection. J Physiol. 2010;588:1139-51.

21. Gadicherla AK, Wang N, Bulic M, Agullo-Pascual E, Lissoni A, De Smet M, et al. Mitochondrial Cx43 hemichannels contribute to mitochondrial calcium entry and cell death in the heart. Basic Res Cardiol. 2017;112:27.

22. Boengler K, Ruiz-Meana M, Gent S, Ungefug E, Soetkamp D, Miro-Casas E, et al. Mitochondrial connexin 43 impacts on respiratory complex I activity and mitochondrial oxygen consumption. J Cell Mol Med. 2012;16:1649-55.

23. Zhang Z, Zhang G, Xu X, Su W, Yu B. mTOR-rictor is the Ser473 kinase for AKT1 in mouse one-cell stage embryos. Mol Cell Biochem. 2012;361:249-57.

24. Kaliszczak M, Trousil S, Ali T, Aboagye EO. AKT activation controls cell survival in response to HDAC6 inhibition. Cell Death Dis. 2016;7:e2286.

25. Rottlaender D, Boengler K, Wolny M, Schwaiger A, Motloch LJ, Ovize M, et al. Glycogen synthase kinase 3beta transfers cytoprotective signaling through connexin 43 onto mitochondrial ATP-sensitive $\mathrm{K}^{+}$channels. Pro Natl Acad Sci USA. 2012;109:E242-51. 
26. Tang LL, Wang JD, Xu TT, Zhao Z, Zheng JJ, Ge RS, et al. Mitochondrial toxicity of perfluorooctane sulfonate in mouse embryonic stem cell-derived cardiomyocytes. Toxicology. 2017;382:108-16.

27. Wu B, Yu H, Wang Y, Pan Z, Zhang Y, Li T, et al. Peroxiredoxin-2 nitrosylation facilitates cardiomyogenesis of mouse embryonic stem cells via XBP-1s/PI3K pathway. Free Radic Biol Med. 2016;97:179-91.

28. Shinozawa T, Furukawa H, Sato E, Takami K. A novel purification method of murine embryonic stem cell- and human-induced pluripotent stem cellderived cardiomyocytes by simple manual dissociation. J Biomol Screen. 2012;17:683-91.

29. Liang $X$, Mei $Y$, Huang $X$, Shen $G$, Zhu D, Yu Y, et al. Junctophilin 2 knockdown interfere with mitochondrium status in ESC-CMs and cardiogenesis of ES cells. J Cell Biochem. 2012;113:2884-94.

30. Rodriguez-Sinovas A, Boengler K, Cabestrero A, Gres P, Morente M, Ruiz-Meana $M$, et al. Translocation of connexin 43 to the inner mitochondrial membrane of cardiomyocytes through the heat shock protein 90-dependent TOM pathway and its importance for cardioprotection. Circ Res. 2006;99:93-101.

31. Valli A, Rosner M, Fuchs C, Siegel N, Bishop CE, Dolznig $H$, et al. Embryoid body formation of human amniotic fluid stem cells depends on mTOR. Oncogene. 2010;29:966-77.

32. Jacinto E, Loewith R, Schmidt A, Lin S, Ruegg MA, Hall A, et al. Mammalian TOR complex 2 controls the actin cytoskeleton and is rapamycin insensitive. Nat Cell Biol. 2004;6:1122-8.

33. Betz C, Stracka D, Prescianotto-Baschong C, Frieden M, Demaurex N, Hall MN Feature Article: mTOR complex 2-Akt signaling at mitochondria-associated endoplasmic reticulum membranes (MAM) regulates mitochondrial physiology. Proc Natl Acad Sci USA. 2013; 110:12526-34.

34. Michela $\mathrm{P}$, Velia V, Aldo $\mathrm{P}$, Ada P. Role of connexin 43 in cardiovascular diseases. Eur J Pharmacol. 2015;768:71-6.

35. Hou S, Zhao MM, Shen PP, Liu XP, Sun Y, Feng JC. Neuroprotective effect of salvianolic acids against cerebral ischemia/reperfusion injury. Int J Mol Sci. 2016;17:1190-200.
36. Kekatpure VD, Dannenberg AJ, Subbaramaiah K. HDAC6 modulates Hsp90 chaperone activity and regulates activation of aryl hydrocarbon receptor signaling. J Biol Chem. 2009;284:7436-45.

37. Li ZY, Li QZ, Chen L, Chen BD, Zhang C, Wang X, et al. HPOB, an HDAC6 inhibitor, attenuates corticosterone-induced injury in rat adrenal pheochromocytoma PC12 cells by inhibiting mitochondrial GR translocation and the intrinsic apoptosis pathway. Neurochem Int. 2016;99:239-51.

38. Yu S, Cai X, Wu C, Liu Y, Zhang J, Gong X, et al. Targeting HSP90-HDAC6 regulating network implicates precision treatment of breast cancer. Int J Biol Sci. 2017;13:505-17.

39. Liu F, Zhao X, Qian Y, Zhang J, Zhang Y, Yin R. MiR-206 inhibits Head and neck squamous cell carcinoma cell progression by targeting HDAC6 via PTEN/AKT/ mTOR pathway. Biomed Pharmacother. 2017;96:229-37.

40. Tao H, Chen YY, Sun ZW, Chen HL, Chen M. Silence of HDAC6 suppressed esophageal squamous cell carcinoma proliferation and migration by disrupting chaperone function of HSP90. J Cell Biochem. 2018;119:6623-32.

c)

Open Access This article is licensed under a Creative Commons Attribution 4.0 International License, which permits use, sharing, adaptation, distribution and reproduction in any medium or format, as long as you give appropriate credit to the original author(s) and the source, provide a link to the Creative Commons license, and indicate if changes were made. The images or other third party material in this article are included in the article's Creative Commons license, unless indicated otherwise in a credit line to the material. If material is not included in the article's Creative Commons license and your intended use is not permitted by statutory regulation or exceeds the permitted use, you will need to obtain permission directly from the copyright holder. To view a copy of this license, visit http://creativecommons. org/licenses/by/4.0/.

(c) The Author(s) 2021, corrected publication 2022 